\title{
An INTEGRAL monitoring of GRS 1915+105 using simultaneous space and ground based instruments
}

\author{
Jérôme Rodriguez*1, Guy Pooley ${ }^{2}$, Diana Hannikainen ${ }^{3}$, Harry J. Lehto ${ }^{4}$, Tomaso \\ Belloni $^{5}$, Marion Cadolle-Bel ${ }^{1}$, Stéphane Corbel ${ }^{6,1}$
}

${ }^{1}$ CEA Saclay, France

${ }^{2}$ Cavendish Laboratory, University of Cambridge, UK

${ }^{3}$ Observatory, University of Helsinki, Finland

${ }^{4}$ Tuorla Observatory, Turku Finland \& Nordita, Denmark

${ }^{5}$ Oss. Astronomico di Brera, Merate, Italy

${ }^{6}$ Université Paris 7, France

E-mail: jrodriguez@cea.fr

\begin{abstract}
We report the results of 3 observations of GRS 1915+105 during which the source is found to show the X-ray dips/spike sequences (cycles). These observations were performed simultaneously with INTEGRAL, RXTE, the Ryle and Nançay radio telescopes. They show the so-called $v, \lambda$ and $\beta$ classes of variability during which a high level of correlated X-ray and radio variability is observed. We study the connection between the accretion processes seen in the X-ray behaviour, and the ejections seen in radio. By observing ejection during class $\lambda$, we generalise the fact that the discrete ejections in GRS 1915+105 occur after the cycles seen at X-ray energies, and identify the most likely trigger of the ejection through a spectral approach to our INTEGRAL data. We show that each ejection is very probably the result of the ejection of a Comptonising medium responsible for the hard X-ray emission seen above $15 \mathrm{keV}$ with INTEGRAL. We study the rapid variability of the source, and observe the presence of Low Frequency Quasi Periodic Oscillations during the X-ray dips. The ubiquity of the former behaviour, and the following ejection may suggest a link between the QPO and the mechanism responsible for the ejection.
\end{abstract}

VI Microquasar Workshop: Microquasars and Beyond September 18-22, 2006

Como, Italy

\footnotetext{
* Speaker.
} 


\section{Introduction}

GRS $1915+105$ is probably the most spectacular high-energy source of our Galaxy. An extensive review on it can be found in Fender \& Belloni (2004). To summarize, GRS 1915+105 is a microquasar hosting a black hole (BH) of $14.0 \pm 4.4 \mathrm{M}_{\odot}$ (Harlaftis \& Greiner 2004), it is one of the brightest X-ray sources in the sky and it is a source of superluminal ejection (Mirabel \& Rodriguez 1994), with true velocity of the jets $\geq 0.9 c$. The source is also known to show a compact jet during its periods of low steady levels of emission (e.g. Fuchs et al. 2003). Multi-wavelength coverage from radio to X-ray has shown a clear but complex association between the soft X-rays and radio/IR behaviour. Of particular relevance is the existence of radio QPO in the range 20-40 min associated with the X-ray variations on the same time scale (e.g. Mirabel et al. 1998). These so called "30-minute cycles" were interpreted as being due to small ejections of material from the system, and were found to correlate with the disc instability, as observed in the X-ray band.

Extensive observations at X-ray energies with RXTE have allowed Belloni et al. (2000) to classify all the observations into 12 separate classes (labeled with greek letters), which could be interpreted as transitions between three basic states (A-B-C): a hard state and two softer states. These spectral changes are, in most classes, interpreted as reflecting the rapid disappearance of the inner portions of an accretion disc, followed by a slower refilling of the emptied region (Belloni et al. 1997).

In the X-ray timing domain, GRS $1915+105$ also shows interesting features, such as the presence of Low or High Frequency Quasi-Periodic Oscillations (LFQPO, HFQPO) whose presence is, as observed in other microquasars, tightly linked to the X-ray behaviour. LFQPOs with variable frequency during classes showing cycles have been reported. Correlations between the frequency and some of the spectral parameters have been pointed out (e.g. Markwardt et al. 1999, Rodriguez et al. 2002a,b). GRS 1915+105 is also one of the first BH systems in which the presence of HFQPOs has been observed, first at $\sim 65-69 \mathrm{~Hz}$, and up to $\sim 170 \mathrm{~Hz}$ (Morgan, Remillard \& Greiner, 1997; Belloni et al. 2006).

The link between the accretion and ejection processes is, however, far from understood and different kinds of model are proposed to explain all observational facts also including the X-ray low $(0.1-10 \mathrm{~Hz})$ frequency QPOs. The monitoring campaign we have performed since late 2002 with INTEGRAL and other instruments (mainly RXTE and the Ryle Telescope, but also Spitzer, Nançay, GMRT, Suzaku; see Ueda et al. these proceedings for the report of the latter) has the aim to try to understand the physics of the accretion-ejection phenomena, including, for the first time, the behaviour of the source seen above $20 \mathrm{keV}$ up to few hundred $\mathrm{keV}$. We report here the results obtained during observations performed during $\mathrm{AO} 2$ and $\mathrm{AO} 3$ showing sequences of $\mathrm{X}$-ray hard dips/soft spikes (the cycles), followed by radio flares. The X-ray spectral results of these observations can be found in Rodriguez et al. (2006), while the full details of these analysis and extended discussions will be presented in a forthcoming paper (Rodriguez et al.in prep.).

\section{Description of the 3 observations}

We focus in this paper on 3 observations respectively taken on October 17-18 2004 (Obs. 1), November 15-16 2004 (Obs. 2), and May 13-14 2005 (Obs. 3). The multiwavelength light curves 


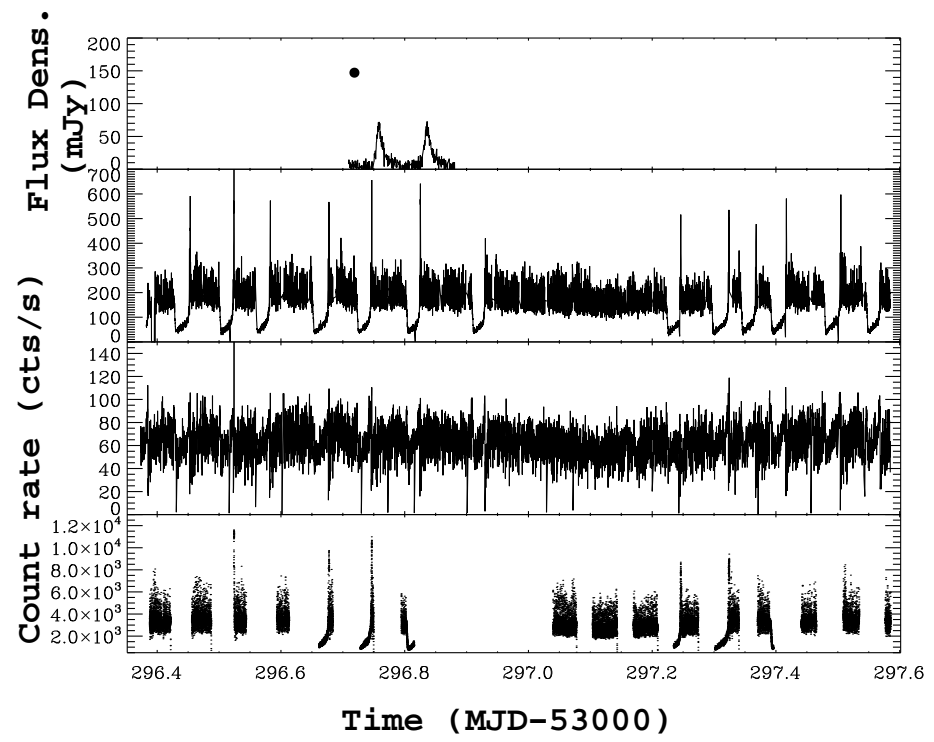

Figure 1: Multiwavelength light curves of GRS 1915+105 on October 17-18 2004 showing two intervals of class $v$ variability. From top to bottom: Ryle (line) at $15 \mathrm{GHz}$, and Nançay (point) at $2.7 \mathrm{GHz}$, JEM-X 3-13 keV, ISGRI 18-100 keV, RXTE 2-60 keV.

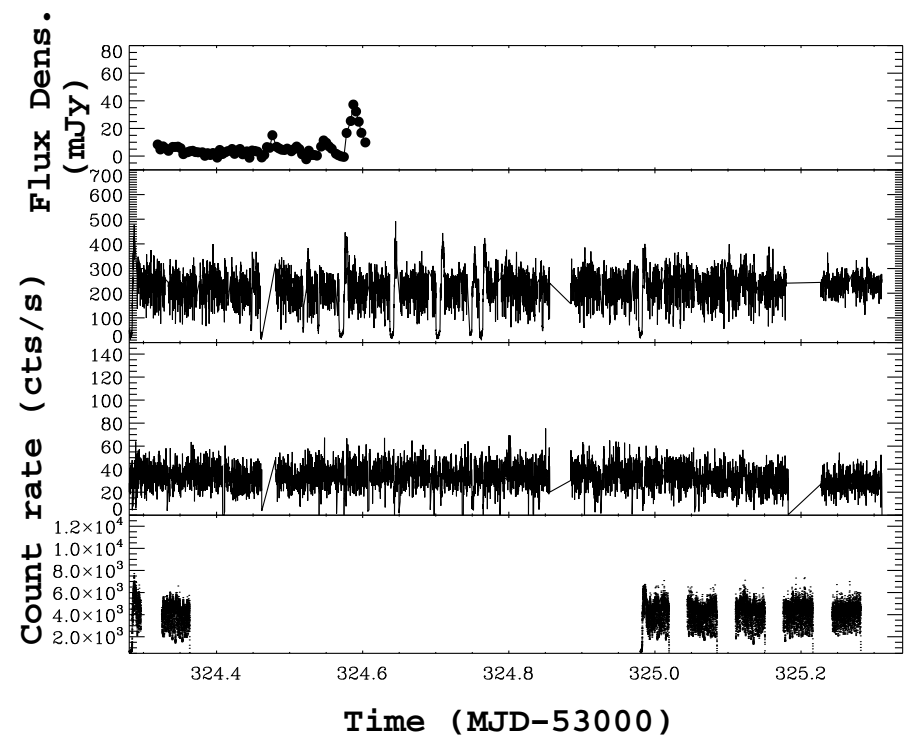

Figure 2: Multiwavelength light curves of GRS 1915+105 on November 2004 during class $\lambda$ variability. The panels are the same as in Fig. 1 with the exception that no Nançay data are available.

are shown in Fig. 1 to 3. The JEM-X light curves show in all cases the occurrences of soft X-ray dips of different duration, followed by a short spike marking the return to a high degree of soft $\mathrm{X}$-ray emission and variability (hereafter cycle). In all the following we focus on the moments of cycle activity and more specifically on the intervals during which we have simultaneous data at radio wavelengths, and/or RXTE data for the timing analysis. This is particularly relevant for Obs.1 and 3 during which transitions between different classes are seen. We, therefore, avoid including in 


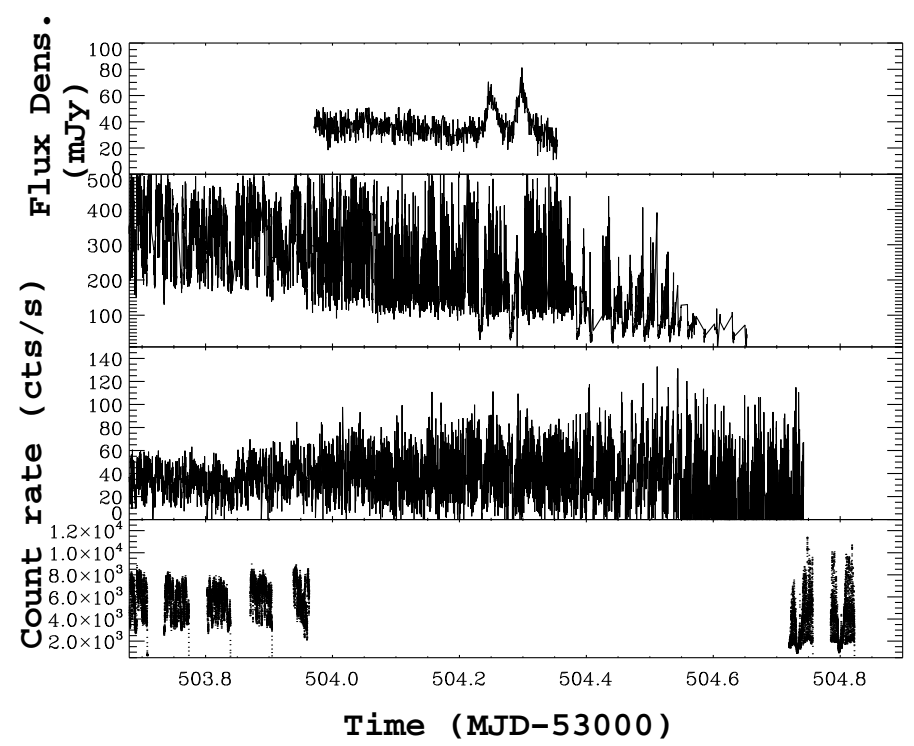

Figure 3: Multiwavelength light curves of GRS $1915+105$ on May 2005 with the appearance of class $\beta$ variability half way through the observation. The panels are the same as in Fig. 2.

our analysis classes for which the radio behaviour is not known. These observations are classified following Belloni et al. (2000) as $v, \lambda$, and $\beta$ type cycle activity for Obs. 1, 2 and 3, respectively. In all three cases we observe at least one radio flare, which is indicative of a small ejection of material (Mirabel et al. 1998), after a cycle. The high flux shown by Nançay at $2.7 \mathrm{GHz}$ during the class $v$ observation suggests that the radio flares follow systematically each cycle, as reported by Klein-Wolt et al. (2002). The presence of radio oscillations is also known in class $\beta$, while no radio/X-ray connection had ever been observed during a class $\lambda$.

\section{X-ray Spectral Analysis}

\subsection{Selection of Good Time Intervals}

In order to study the spectral evolution of GRS 1915+105 through the cycle and the possible origin of the radio ejection, we divided the cycles into different intervals from which JEM-X and ISGRI spectra were accumulated. Each cycle was divided in, at least, 3 intervals based on the soft X-ray count rate and the 3-13/18-100 keV hardness ratio. The intervals are defined as follow:

In each cycle four distinct intervals can be distinguished. The soft X-ray dip, having a hard spectrum (interval A), a short precursor spike (interval B), a (short) following dip (interval C) and the main spike (interval $\mathrm{D}$ ), the last 3 having soft spectra. In class $\lambda$ and $\beta$ the observation of radio oscillations (Klein-Wolt et al. 2002), i.e. the occurrence of radio flares after each cycle, allow us to further accumulate together all cycles before the fitting to increase the statistics. In Class $\lambda$, occurrences of a pairs of cycles at some moments can happen, while at other intervals the cycles are isolated. Since no X-ray/radio connection had ever been observed in this class before, we extracted spectra from the unique cycle that is followed by an ejection. The same sequence of $\mathrm{A}, \mathrm{B}, \mathrm{C}$, 


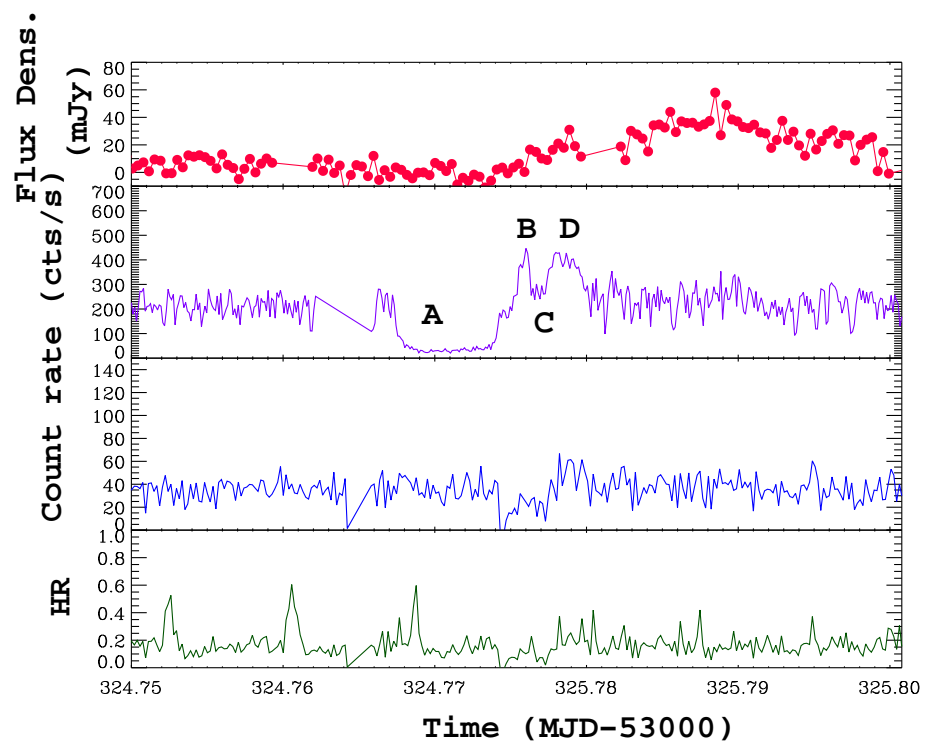

Figure 4: A section on the X-ray cycle followed by the radio flare from class $\lambda$. The 4 intervals from which the spectra were accumulated (see text) are indicated. From top to bottom, the panels respectively represent the Ryle light curve, the 3-13 keV JEM-X light curve, the 18-100 keV ISGRI light curve, and the 18-100/3-13 keV hardness ratio.

D interval can also be identified (Fig. 4). Note the A, B, C, D labels for the different intervals are not related to the spectral states (A, B and C) identified by Belloni et al. (2000). In order to be able to compare the different spectra, we fitted them all with the same model consisting of a thermal component (ezdisk) and a Comptonised one (comptt). Fig. 5 shows, as an example, the sequence of spectra from class $\lambda$.

\subsection{Results}

In all cases the evolution of the source through the cycles is more prononced in the soft X-rays (Fig. 1 to 3). At first sight we could think that the variations are then caused by changes in the accretion disc rather than in the Comptonised component. When fitting the different spectra with physical models, however, it seems to be the contrary. In class $v$ and $\lambda$ the evolution through A to $\mathrm{D}$ translates into an apparent approach of the accretion disc to the black hole, with an increasing temperature. Interestingly between $\mathrm{B}$ and $\mathrm{C}$, the disc parameters are compatible with being constant within the errors. We calculated the $3-50 \mathrm{keV}$ unabsorbed fluxes of the two spectral components in the 4 intervals. While the flux from the disk increases through the whole sequence, that of the Comptonised component is not that regular. It first increases from $v_{A}$ to $v_{B}$ before decreasing by a factor of 2.5 in $v_{C}$ (reaching $1.0 \times 10^{-8} \mathrm{erg} \mathrm{cm}^{-2} \mathrm{~s}^{-1}$ ), and slowly recovers in $v_{D}\left(1.5 \times 10^{-8}\right.$ erg $\mathrm{cm}^{-2} \mathrm{~s}^{-1}$ ). It follows similar evolution in $\lambda$ (this is exemplified by Fig. 5), with in particular a reduction by a factor of $\sim 11$ between $\lambda_{B}$ and $\lambda_{C}$ (from $2.2 \times 10^{-8} \mathrm{erg} \mathrm{cm}^{-2} \mathrm{~s}^{-1}$ to $0.19 \times 10^{-8}$ erg $\mathrm{cm}^{-2} \mathrm{~s}^{-1}$, when leaving all spectral parameters free to vary), with a lower limit of 2.7 (if the disc temperature is frozen to the same value as in $\lambda_{B}$ ). Again after $\lambda_{C}$ the Comptonised component slightly recovers $\left(1.32 \times 10^{-8} \mathrm{erg} \mathrm{cm}^{-2} \mathrm{~s}^{-1}\right)$.

The spectral behaviour of the source during class $\beta$ has been extensively studied in the past. 

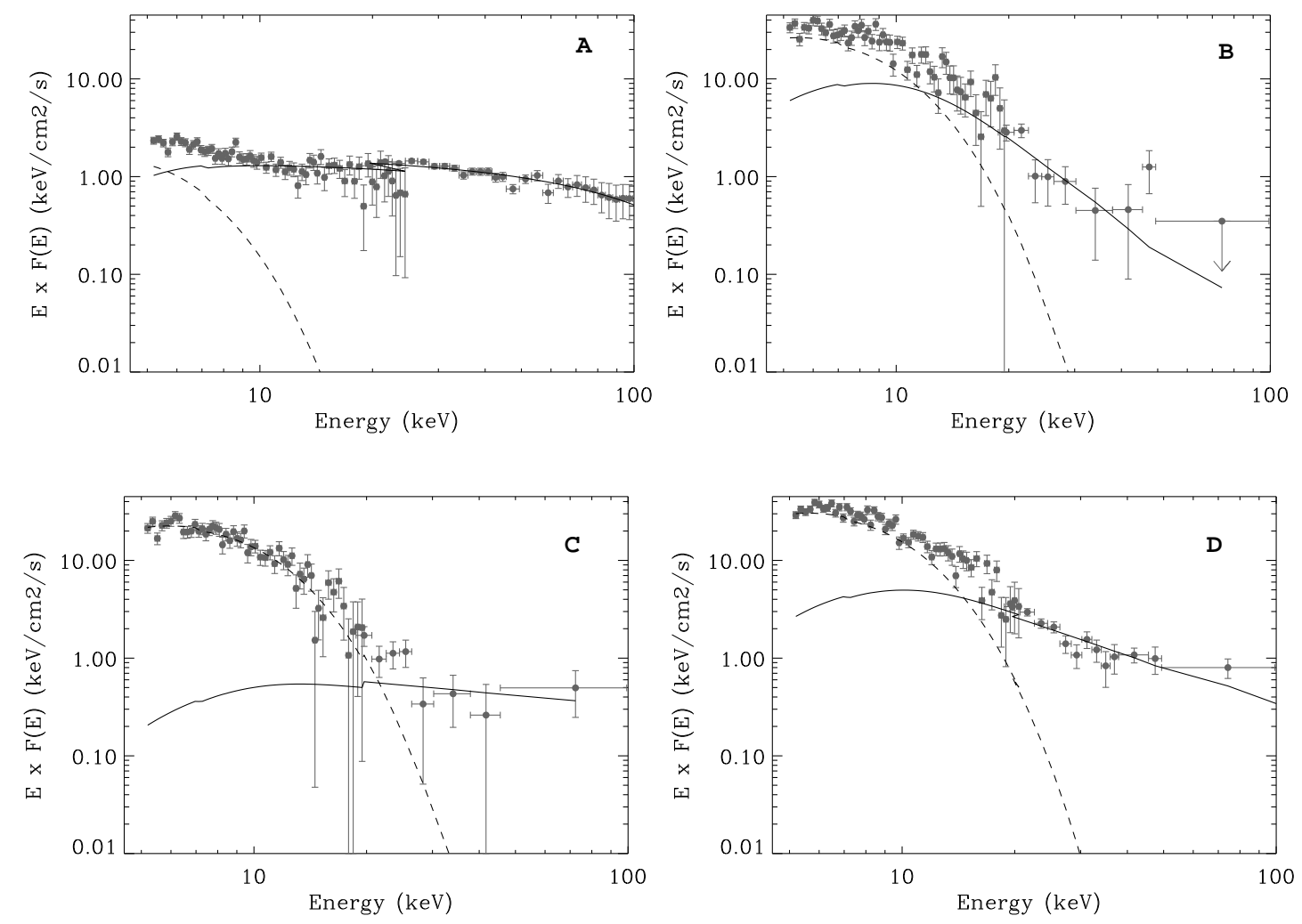

Figure 5: The 4 spectra and best models superimposed from class $\lambda$. The individual components are also shown. The disc component is represented by the dashed line, while the Comptonised component is represented by the solid line. The evolution through the dips is quite obvious, with in particular a spectacular drop in the Comptonised component between B and C.

It is interesting to note that, as for the previous two classes, the disc temperature slowly increases during the dip, even after the spike (Swank et al. 1998), while the inner radius remains small, indicating the disc is close to the compact object. The transition, after the spike, to state A (Belloni et al. 2000) the very soft spectral state of GRS 1915+105, suggest that the spectacular changes occurring at the spike are related to the Comptonised component.

\section{Timing analysis: Low Frequency QPOs}

Although most of the cycles seen with RXTE were not simultaneous with the cycles followed by radio ejections, we produced dynamical power spectra of the cycles with the view to study whether or not the soft X-ray dips were associated with a LFQPO of variable frequency, as has been seen in class $\beta$, and $\alpha, v$ and $\theta$ (Rodriguez et al.2002a,b; Vadawale 2003). The dip preceding the radio ejection during class $\lambda$ was unfortunately not covered by RXTE. Assuming the same pattern repeats during all dips, we extracted a dynamical power spectrum from the unique sequence of dips covered by RXTE. This is shown in Fig. 6 together with the associated light curve. In the other classes i.e. $\lambda$ and $\beta$, the presence of LFQPOs with variable frequencies during the dips are 


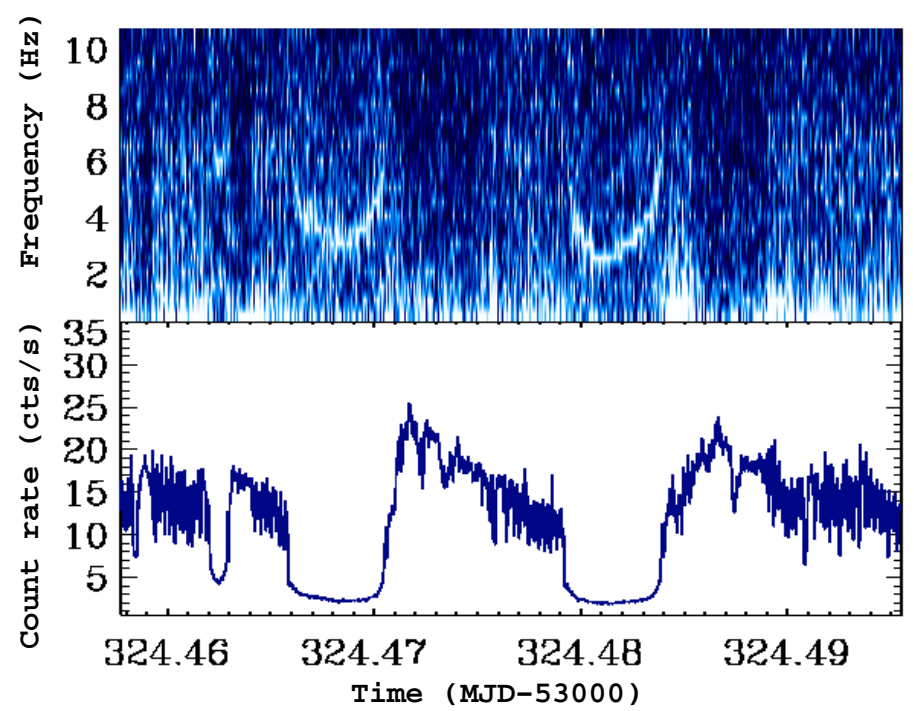

Figure 6: Dynamical power spectrum (top) and RXTE/PCA light curve (bottom) on the unique 2 cycles from class $\lambda$ covered by $R X T E$.

confirmed by our analysis (not shown here).

As in the other classes showing soft X-ray dips, a strong QPO with variable frequency appears during the dip (Fig. 6). Here, however, the frequency seems less tightly correlated to the X-ray count rate, since while during the dips the count rate remains rather constant, the frequency of the QPO is less stable.

\section{Discussion and conclusion}

We have presented observations of GRS 1915+105 made in simultaneity with INTEGRAL, $R X T E$, the Ryle telescope and in one occasion the Nançay telescope. These observations belong to classes $v, \lambda$, and $\beta$, which have the characteristic of showing sequences of soft X-ray dips followed by high level of $\mathrm{X}$-ray emission with high variability, sequences we referred to as cycles. While in some of these classes ejections (observed in the radio domain) had been observed to follow to recovery to a high level of X-ray emission (Mirabel et al. 1998), the observation of a radio flare during the class $\lambda$ observation is, to our knowledge, the first ever reported. This allows us to generalise the fact that there is always an ejection following a cycle. The duration of the dip during class $\lambda$ is $500 \mathrm{~s}$, while it is as low as $330 \mathrm{~s}$ during class $\beta$. This duration is in agreement with the results of Klein-Wolt et al. (2002) who observed that for an ejection to occur a longer than 100s hard state must occur.

Detailed inspection of the cycles shows that the sequences are not as simple as it seems at first sight, but that in all cases a precursor spike occurs prior to the recovery to the high X-ray level. Our spectral analysis of the different portions of the cycles shows that the same evolution roughly occurs during the cycles from different classes. During the X-ray dip the source is in its hard state (or so-called C state for GRS 1915+105 Belloni et al. 2000). The precursor spikes correspond 
to a simultaneous softening of the source spectrum and an increase in the flux of the two main spectral components, the disc and the corona. The short dip immediately following the precursor corresponds to the disappearance of the corona in the two cases we analysed in detail. The same had been observed in the case of class $\beta$ (Chaty 1998). Given the observation of ejected material soon after, we conclude that:

\section{1) The ejected material is the coronal material}

\section{2) The true moment of the ejection is the precursor spike}

It has to be noted that Chaty (1998), Rodriguez et al. (2002b), Vadawale (2003) had come to similar conclusions during other classes. Another point compatible with this scenario is that if we estimate the lag between the radio and the X-ray, we obtain $0.28 \mathrm{~h}$ and $0.26 \mathrm{~h}$ during class $v, 0.31 \mathrm{~h}$ in class $\lambda$, and 0.29 and $0.34 \mathrm{~h}$ during class $\beta$, therefore the delay is comparable in all classes. Interestingly the same conclusion had been drawn in another microquasar XTE J1550-564. During its 2000 outburst (Rodriguez et al. 2003) have shown that the discrete ejection following the maximum of the X-ray emission was compatible with the ejection of the coronal medium.

Although the physical properties of the dips are not exactly the same, some other similarities raise interesting questions. The timing analysis of the classes showing cycles all show the presence of a transient LFQPO appearing during the dip, and with a variable frequency which may be somehow correlated to the X-ray flux (Swank et al. 1998; Rodriguez et al. 2002a,b; Vadawale 2003). The presence of LFQPO during hard states is quite common, and may be a signature of the physics occurring during the accretion and the ejection of matter, since a steady compact jet is usually associated with this state. In the case of the cycles, a discrete ejection seems to take place at the end of each cycle. The presence of LFQPO during the dip may, again, indicate that the QPO phenomenom and the accretion-ejection physics are linked.

Recently Tagger et al. (2004) have proposed a "magnetic flood" scenario in trying to explain the occurrence of dips and ejections, based on a magnetic instability, the Accretion Ejection Instability (AEI, Tagger \& Pellat 1999). This instability has the effect of transporting angular momentum and energy from the inner region of the disc, and emitting them perpendicularly and directly into the corona. A strong observational signature of this AEI is the presence of LFQPOs. In this framework the X-ray dip would correspond to the appearance of the AEI (equivalent to a transition to a hard state with appearance of LFQPO), and the ejection could be due to a reconnection event in the inner region of the disc (Tagger et al. 2004). An effect of the latter would be to blow the corona and would end observationally as an ejection. Although none of our results brings proof of such a scenario, and that other models exist, this scenario is compatible with our results. In particular with the generalisation of the ejection during all classes with dips, the relative similarities between the dips of all classes, both at radio and X-ray energies, and the constant presence of LFQPO during the dips, we feel that this model is a very promising one.

\section{Acknowledgments}

These results are presented on behalf of a much larger collaboration whose members the authors deeply thank. JR is extremely grateful to J. Chenevez, and C.-A. Oxborrow for their precious help with the JEM-X data reduction, and M. Tagger for a careful reading of an early version of this paper. JR acknowledges E. Kuulkers and E. Smith and more generally the INTEGRAL and RXTE 
planning teams for their great efforts to have both satellites observing GRS $1915+105$ and IGR J19140+0951 at the same time.

\section{References}

[1] Belloni, T., Mendez, M., King, A. R., et al. 1997, ApJ, 488, 109

[2] Belloni, T., Klein-Wolt, M., Mendez, et al. 2000, A\&A, 355, 271

[3] Belloni, T., Soleri, P., Casella, P., Méndez, M., Migliari, S. 2006, MNRAS 369, 305.

[4] Chaty, S. 1998, Thèse de Doctorat "Etude multi-longueur d'onde du microquasar GRS 1915+105 et de sources binaires.de haute énergie de la Galaxie"

[5] Fender, R.P. \& Belloni, T. 2004, ARA\&A, 42, 317

[6] Fuchs, Y., Rodriguez, J., Mirabel, F., et al. 2003, A\&A, 409, L35.

[7] Harlaftis, E.T \& Greiner, J., 2004, A\&A, 414, 13.

[8] Klein-Wolt, M., Fender, R. P.; Pooley, G. G., et al. 2002, MNRAS, 331, 745

[9] Markwardt, C.B., Swank, J. H., Taam, R. E. 1999, ApJ, 513, 37.

[10] Mirabel, I.F. \& Rodríguez, L.F. 1994, Nature, 371, 46

[11] Mirabel, I.F., Dhawan, V., Chaty, S., et al. 1998, A\&A, 330, L9

[12] Morgan, E.H., Remillard, R.A \& Greiner, J. 1997, ApJ, 482, 993

[13] Rodriguez, J., Durouchoux, P., Tagger, M., et al., 2002a, A\&A, 386, 271

[14] Rodriguez, J., Varnière, P., Tagger, M., Durouchoux, P. 2002b, A\&A, 387, 487

[15] Rodriguez, J., Corbel, S., \& Tomsick, J.A., 2003, ApJ, 595, 1032

[16] Rodriguez, J., Pooley, G., Hannikainen, D.C., Lehto, H.J. 2006, proceedings of the 6th INTEGRAL Workshop, ESA SP622.

[17] Swank, J.H., Chen, X., Markwardt, C., Taam, E. 1998 proceedings of the conference "Accretion Processes in Astrophysics: Some Like it Hot", eds. S. Holt and T. Kallman

[18] Tagger, M. \& Pellat, R. 1999, A\&A, 349, 1003

[19] Tagger, M., Varnière, P., Rodriguez, J. \& Pellat, R. 2004, ApJ, 607, 410

[20] Vadawale, S.V., Rao, A.R., Naik, S., Yadav, J.S., Ishwara-Chandra, C.H., Pramesh Rao, A. and Pooley, G.G. 2003, ApJ, 597, 1023 